\title{
Transcriptome analysis reveals that Müllerian inhibiting substance regulates signaling pathways that contribute to endometrial carcinogenesis
}

\author{
YOUN JEE CHUNG ${ }^{1 *}$, HYUN JUNG KIM ${ }^{1 *}$, SANG HO PARK ${ }^{1}$, JOO HEE YOON ${ }^{1}$, MEE RAN KIM ${ }^{1}$, \\ SUK WOO NAM ${ }^{2}$, DAVID T. MacLAUGHLIN ${ }^{3}$, PATRICIA K. DONAHOE ${ }^{3}$ and JANG HEUB KIM ${ }^{1}$ \\ Departments of ${ }^{1}$ Obstetrics and Gynecology, ${ }^{2}$ Pathology, College of Medicine, The Catholic University \\ of Korea, Seoul 137-701, Republic of Korea; ${ }^{3}$ Pediatric Surgical Research Laboratories, \\ Massachusetts General Hospital, Harvard Medical School, Boston, MA 02114, USA
}

Received December 23, 2014; Accepted February 9, 2015

DOI: $10.3892 / \mathrm{ijo} .2015 .2920$

\begin{abstract}
Müllerian inhibiting substance (MIS) has been shown to inhibit growth of a number of tumors in vitro and/or in vivo, but the downstream pathways which it regulates are not fully understood. In the present study we show that MIS type II receptor was highly expressed in AN3CA cells, a cell line derived from human endometrial cancer cell in which MIS-treatment caused a reduction of cell viability, and induced cellular apoptosis and genes involved cell cycle arrest. To understand the genome-wide effects of MIS on gene regulation, we performed serial gene expression analyses from 0 to $96 \mathrm{~h}$ at $24 \mathrm{~h}$ intervals after treating AN3CA cells with MIS. Transcriptomic analysis of molecular changes induced by MIS identified 2,688 differentially expressed genes that were significantly up- or down-regulated during the $96 \mathrm{~h}$ study period. When the 2,688 differentially expressed genes were mapped to known biological processes, Wnt-, cancer-, proteolysis-, cytoskeleton-, cell cycle-, apoptosis-, and MAPK-signaling pathways emerged as the functions most significantly changed by MIS in AN3CA cells. Furthermore, western blot analysis validated that protein expression of cell cycle inhibitory genes, apoptotic protease activating factor-1 (APAF-1), $\beta$-catenininteracting protein (ICAT), Rb related protein 130 (p130), and inhibitor of disheveled Dvl and Axin complex (IDAX), were gradually increased over the time of the study, whereas downstream cell cycle activating genes, cyclin-dependent
\end{abstract}

Correspondence to: Professor Jang Heub Kim, Department of Obstetrics and Gynecology, Seoul St. Mary's Hospital, College of Medicine, The Catholic University of Korea, 505 Banpo-dong, Seocho-gu, Seoul 137-140, Republic of Korea

E-mail: janghkim@catholic.ac.kr

${ }^{*}$ Contributed equally

Key words: Müllerian inhibiting substance, AN3CA cell line, transcriptome analysis, Wnt signaling pathway, cell cycle, apoptosis kinase 2 (CDK2) and phospho-c-Jun were downregulated in MIS-treated AN3CA cells. These transcriptome analyses support previous observations that MIS functions as a tumor suppressor, potentially by regulating signaling pathways that could contribute to endometrial carcinogenesis, and indicating that MIS should be considered as a potential treatment for endometrial cancer.

\section{Introduction}

Endometrial cancer is the most common invasive gynecologic cancer in the Western world and the fourth most common cancer in women after breast, lung and colorectal cancers (1). Endometrial cancer has been divided into two different types on the basis of distinct histology and clinical outcomes. Most endometrial cancers are type I (approximately 75\%) estrogendependent adenocarcinomas with endometrioid morphology. Type I endometrial cancers are low-grade, early stage cancers that usually develop in perimenopausal women and coexist with, or are preceded by, complex and atypical endometrial hyperplasia, and have an excellent prognosis (2). In contrast, type II endometrial cancers are of poorly differentiated endometrioid and serous histologies that occur mainly at an older age and are associated with a lower 5-year survival rate (3). The definite role of estrogen in the development of most endometrial cancers is established; any factor that increases exposure to unopposed estrogen increases the risk for endometrial cancer. Endometrial cancers are characterized by various genetic alterations. Type I endometrial cancer harbors altered PI3K/AKT/mTOR signaling pathway constituents (4). Type II endometrial cancer have loss of function mutations in p53 (5) and gain of function mutations in epidermal growth factor receptor 2 (HER-2) (6).

Most gynecologic tumors originate from Müllerian ducts, which develop into the Fallopian tubes, uterus, cervix, proximal vagina, and from the surface epithelium of the ovary. Müllerian inhibiting substance (MIS), also known as anti-Müllerian hormone (AMH), has long been known for causing the regression of the embryonic Müllerian duct. MIS has been shown to inhibit tumor growth in vitro and in vivo, 
but its downstream regulated genes have not been fully elucidated. MIS initiates its effect by binding to the MIS type I and type II receptors (7). Several studies showed that MIS exerts antiproliferative and apoptotic effects on gynecologic malignancies such as cervical and ovarian cancers $(8,9)$. In ovarian cancer, the MIS caused a suppressive effect on Cyclin Dependent Kinases (CDKs) and the E2F transcription/ dimerization partner (E2F/DP1) complex, with transcriptional enhancement of certain histone deacetylases, which act as co-suppressors of the E2F/DP complex (9). In cervical cancer, MIS suppresses cell division by inducing an increase in the expression of CDK inhibitors such as $16^{\text {INK4a }}$ which results in a decrease in activity of the CDK complex leading to inhibition of E2F activity (8). Since endometrial cancer is also a tumor of Müllerian duct origin and prior studies (10) have reported antitumor properties of MIS against endometrial carcinoma cell lines, we sought to understand the molecular drivers of growth inhibition or apoptosis in endometrial cancer. To do so, we undertook transcriptome scans of AN3CA cells treated with MIS. Analysis of the differentially expressed genes that were further validated by western analysis indicated that MIS could elicit its tumor suppressive effects on endometrial cancer cells through control of components of pathways on which endometrial cancer progression is dependent.

\section{Materials and methods}

Endometrial cancer cell culture. The human endometrial cancer cell line, AN3CA (American Type Culture Collection, Manassas, VA, USA) was grown in Eagle's minimal essential medium (Gibco, Grand Island, NY, USA) containing 10\% Fetal Bovine Serum (FBS), $100 \mathrm{U} / \mathrm{ml}$ penicillin and $100 \mathrm{U} / \mathrm{ml}$ streptomycin, and incubated at $37^{\circ} \mathrm{C}$ in humid air with $5 \%$ $\mathrm{CO}_{2}$. All experiments were done on low passage cells that were cycled into a proliferative phase and harvested at $80 \%$ confluency.

Immunohistochemical analysis of MIS Type II receptor. Immunohistochemical analysis to detect MIS RII expression was performed using the Invitrogen Histostain Plus AEC kit (Invitrogen, Carlsbad, CA, USA) according to the manufacturer's instructions. Cultured AN3CA cells were harvested and $200 \mu \mathrm{l}$ of a $1 \times 10^{5}$ cell/ml suspension was centrifuged with Cytospin (Thermo Electron Corp., Cheshire, UK) at $1000 \mathrm{rpm}$ for $5 \mathrm{~min}$ and attached to Probe-on-plus-slides. These slides then were treated with $3 \% \mathrm{H}_{2} \mathrm{O}_{2}$ for $5 \mathrm{~min}$ to eliminate endogenous peroxidase activity and washed three times with Tris-buffered saline with $0.1 \%$ Tween-20 (T-TBS). After treatment with donkey serum (Invitrogen) for 30 min to block non-specific protein binding, the slides were incubated with rabbit polyclonal anti-human MIS type II receptor antiserum (Massachusetts General Hospital, Boston, MA, USA) (11) at $4^{\circ} \mathrm{C}$ overnight. The slides were rinsed in T-TBS three times, and incubated with biotinylated anti-rabbit IgG (Invitrogen) for $30 \mathrm{~min}$. After another three T-TBS rinses, the streptavidin HRP detection system (Invitrogen) was applied to the slides for $30 \mathrm{~min}$ to induce the biotin-avidin binding reaction. The slides were treated with 3-amino-9-ethylcarbazole (AEC, Invitrogen) for $10 \mathrm{~min}$ at room temperature, counterstained with hematoxylin, then mounted with glycerol gel.
Methylthiazoletetrazolium (MTT) assay for cell viability. Three thousand cells/well low passage cells that were subcultured three times then harvested at $80 \%$ confluency were seeded in 96-well plates in Dulbecco's modified Eagle's medium (DMEM) with 10\% FBS. After $24 \mathrm{~h}$ the cells were exposed to $71.4 \mathrm{nM}$ MIS for $0,24,48,72$ or $96 \mathrm{~h}$, or the equivalent volume of phosphate buffered saline (PBS, $\mathrm{pH} 7.4$ ) as a vehicle control. The treated cells were washed with PBS and $100 \mu \mathrm{l}$ of MTT solution [5 mg/ml MTT stock (Roche Molecular Biochemicals, Mannheim, Germany) in PBS diluted to $1 \mathrm{mg} / \mathrm{ml}$ with $10 \% \mathrm{DMEM}$ ] was added to each well. Cells were incubated for $4 \mathrm{~h}$ at $37^{\circ} \mathrm{C}$ at the end of which $200 \mu \mathrm{l}$ DMSO was added for $30 \mathrm{~min}$ at room temperature in the dark. Optical densities at $550 \mathrm{~nm}$ were measured using an ELISA plate reader (Bio-Tek Instruments, Winooski, VT, USA).

Cell cycle analysis. AN3CA cells managed as above were exposed to $71.4 \mathrm{nM}$ MIS (Massachusetts General Hospital) (12) for $0,24,48$ or $72 \mathrm{~h}$, or the equivalent volume of PBS ( $\mathrm{pH} 7.4$ ) as a vehicle control and the treated cells were collected by trypsinization. The cells were fixed with $100 \%$ methanol and stored for $30 \mathrm{~min}$ at $20^{\circ} \mathrm{C}$. The cells were washed with PBS, centrifuged, resuspended in $1 \mathrm{ml}$ DNA staining solution (20 $\mu \mathrm{g} / \mathrm{ml}$ propidium iodide, $200 \mu \mathrm{g} / \mathrm{ml}$ DNase free RNase), incubated in the dark at $37^{\circ} \mathrm{C}$ for $30 \mathrm{~min}$, and then analyzed on a FACSVatage SE Flow Cytometer (Becton Dikison, San Jose, CA, USA). The forward scatter and red fluorescence $>600 \mathrm{~nm}$ were measured and the results analyzed using CellQuest software and Modfit LT 3.0 program (Verity Software House, Topsham, ME, USA).

Annexin $V$ analysis. AN3CA cells managed as above were treated with $71.4 \mathrm{nM}$ MIS for the same intervals then harvested and stained for Annexin $\mathrm{V}$ and propidium iodide (PI) using the Annexin V-FITC Apoptosis Detection kit I (BD Biosciences, San Diego, CA, USA) according to the manufacturer's protocol. Brielfy, following drug treatment, $10^{5}$ cells were pelleted, washed once with PBS, and resuspended in $100 \mu \mathrm{l}$ of binding buffer [10 mM HEPES (pH 7.4), $150 \mathrm{mM} \mathrm{NaCl}, 5 \mathrm{mM}$ potassium chloride, $1 \mathrm{mM} \mathrm{MgCl}_{2}$, and $2 \mathrm{mM}$ calcium chloride]. Subsequently, $5 \mu \mathrm{l}$ of Annexin V-FITC and PI was added to the cells which were then incubated for $15 \mathrm{~min}$ at room temperature in the dark. After incubation, $400 \mu \mathrm{l}$ of binding buffer was added to the stained cells which were analyzed using a FACSVatage SE flow cytometer (Becton Dickinson). Data analysis was conducted using CellQuest software.

RNA isolation and gene profiling. Total RNA was extracted from the AN3CA cells managed as above with TRIzol reagent (Invitrogen), and the purity and yield of the RNA were determined spectrophotometrically. The quality of total RNA was analyzed by using the RNA StdSens Chips on the Experion ${ }^{\mathrm{TM}}$ system (Bio-Rad,Hercules, CA, USA). Microarray analysis was performed by using Human HT-12 v4 Expression BeadChip (Illumina, Inc., San Diego, CA, USA). The RNA was processed by using the Illumina RNA Amplification kit (Ambion, Inc., Austin, TX, USA) according to the manufacturer's instructions starting with $1.1 \mu \mathrm{g}$ total RNA. Resulting biotin-labeled cRNA was recovered and purified with RNeasy kit (Qiagen, Valencia, CA, USA), hybridized to the chips, and fluorescently tagged 
and scanned by using Illumina BeadStation (Illumina, Inc.) according to the manufacturer's protocol. All arrays were run in the array core facility of the Functional RNomics Research Center at the Catholic University of Korea.

Microarray data processing and analysis for gene expression. BeadStudio (version 3.0) was used for data acquisition and calculation of signal values on Illumina expression microarray. Normalization of microarray data and hierarchical clustering were performed by using GenPlex ${ }^{\mathrm{TM}}$ (version 3.0). Sets of differentially expressed genes were identified by a parametric test (Welch's t-test). A threshold P-value in combination with fold change was applied. Expression profiles of the gene sets with a fold change regulation of more than 1.3 -fold and $\mathrm{P}<0.05$ were examined to find the differentially expressed genes. Hierarchical clustering was performed by using Cluster and TreeView 2.3 (Stanford University). Euclidean correlation, median centering, and complete linkage were applied during all clustering applications.

Western blot analysis. Proteins from $10 \mu \mathrm{g} / \mathrm{ml}$ of MIS treated cells were harvested in radioimmunoprecipitation assay (RIPA) buffer $(150 \mathrm{mM} \mathrm{NaCl}, 1 \% \mathrm{NP}-40,0.5 \%$ sodium deoxycholate, $0.1 \%$ SDS, $50 \mathrm{nM}$ Tris- $\mathrm{HCl}$ ) with $1 \mu \mathrm{M}$ phenylmethylsulfonyl fluoride and protein concentration was determined by bicinchoninic acid protein assay reagent (Thermo Scientific, Waltham, MA, USA). Equal amounts of protein were separated on sodium dodecyl sulfate $(8-12 \%$ gel) polyacrylamide gels (50 $\mu \mathrm{g}$ per lane), and transferred to polyvinylidene difluoride membrane. The blots were blocked in TBS-T $(20 \mathrm{mM}$ Tris-HCl, pH 7.6, $137 \mathrm{mM} \mathrm{NaCl}, 0.1 \%$ Tween-20) containing $5 \%$ powdered milk for $1 \mathrm{~h}$ and then incubated in $1 \%$ milk TBS-T with the primary antibodies at $4^{\circ} \mathrm{C}$ overnight. Apaf- 1 (sc-8339, Santa Cruz Biotechnology, Santa Cruz, CA, USA), caspase-3 (9668, Cell Signaling Technology, Inc., Boston, MA, USA), CDK2 (sc-6248, Santa Cruz Biotechnology), ICAT (sc-99240, Santa Cruz Biotechnology), Idax (sc-164631, Santa Cruz Biotechnology), PARP (9542, Cell Signaling Technology, Inc.), phospho-c-Jun (3270s, Cell Signaling Technology, Inc.), p107 (sc-318, Santa Cruz Biotechnology) and p130 (sc-317, Santa Cruz Biotechnology) were used at a 1:200 dilutions. Blots were then washed three times with $1 \%$ milk TBS-T, incubated with the corresponding horseradish peroxidaseconjugated secondary antibody, and detected using the Pierce ECL western blotting substrate (Thermo Scientific).

Statistical analysis. Results are presented as mean \pm SD of replicate analyses and are either representative of, or inclusive of, at least three independent experiments. Statistical comparisons between two experimental groups were performed using Student's t-test (paired) whilst multiple group comparisons were performed using analysis of variance (ANOVA). Data were regarded as statistically significant when $\mathrm{P}<0.05$.

MTT results are presented as percentage of control, which was calculated using the following equation: (mean absorbance of treated cells/mean absorbance of control cells) $\mathrm{x} 100$. Data are expressed as mean $\pm \mathrm{SD}$ from nine independent experiments, each with replicates of three. A P-value $<0.05$ was considered statistically significant when compared with corresponding vehicle control cells.
Cell cycle distributions after exposure of AN3CA endometrial cancer cells to MIS are presented as histograms of the mean \pm SD from three independent experiments. Annexin V analysis was done to evaluate apoptosis. Quadrant rectangular dot grams from a representative of three independent experiments were analyzed.

\section{Results}

Treatment results of Müllerian inhibiting substance in growth retardation and cellular apoptosis of AN3CA endometrial cancer cells. It has been well established that proliferation of ovarian, cervical and breast cancer cells are suppressed by MIS treatment, and many of these studies mentioned the potential of MIS as a treatment of MIS-receptor expressing tumors. Therefore, before initiating experiments on the regulatory roles of MIS in endometrial cancer cells, it was necessary to confirm expression of the MIS receptor in AN3CA cells. To this end, we performed immunohistochemical staining with an anti-MIS type II receptor (MISR II) antibody on AN3CA cells which detected strong expression on their cell surface (Fig. 1A).

Next, to investigate the antitumor effects of MIS on endometrial cancer cells, AN3CA cells were treated with MIS at the indicated concentration for $0,24,28,72$ and $96 \mathrm{~h}$, and MTT assays were performed. As shown in Fig. 1B, MIS treatment on AN3CA cells resulted in a 30\% reduction of tumor cell growth ( $n=3, P<0.05$, using Student's $t$-test) resulting from cell cycle arrest or apoptosis. Cell cycle analysis of PI-stained cells using flow cytometry at $72 \mathrm{~h}$ after MIS treatment (Fig. 1C) detected a slight $4.85 \%$ increase in cells in the G1 phase, with a concomitant decrease in S phase and G2/M phase by 3.88 and $4.61 \%$, respectively, compared to controls (non-treatment) $(\mathrm{n}=3, \mathrm{P}<0.05$, using Student's t-test). Flow cytometric analysis at $72 \mathrm{~h}$ of Annexin V stained AN3CA cells (Fig. 1D), however, showed a statistically significant increase $(n=3, P<0.0001$, using Student's t-test) in early cellular apoptosis of $14.37 \%$ (from $2.31 \pm 0.18 \%$ at $0 \mathrm{~h}$ to $16.68 \pm 1.21 \%$ at $72 \mathrm{~h}$ ) and in late cellular apoptotic and necrotic cells of $6.49 \%$ (from $0.04 \pm 0.03 \%$ at $0 \mathrm{~h}$ to $6.53 \pm 0.75 \%$ at $72 \mathrm{~h}$ ) (Fig. 1D). These results suggest that MIS exerts its antitumor effects on endometrial cancer cells by causing significant cellular apoptosis.

Characterization of molecular and functional signatures after MIS treatment of endometrial cancer cells. To identify a molecular signature induced by MIS, serial gene expression analysis was conducted. AN3CA cells were continuously treated with MIS for up to $96 \mathrm{~h}$, and the RNAs were harvested for microarray analysis at multiple time points from $6 \mathrm{~h}$. In Fig. 2A, 11,470 genetic elements, which passed the minimum selection and filtering criteria, are shown in unsupervised hierarchical clustering. Mathematical comparisons of genes between $0 \mathrm{~h}$ and the $6-96 \mathrm{~h}$ time points were performed and 2,688 genes showing at least 1.3-fold changes (Fig. 2B) were visualized as a heat map where red indicates that expression levels of genetic elements that are higher after MIS treatment and green indicates that expression levels of genetic elements that are lower than the mean value of no treatment.

To derive insight into the molecular pathways in which these 2,668 genes participate, we used the pathway tool in the Kyoto 

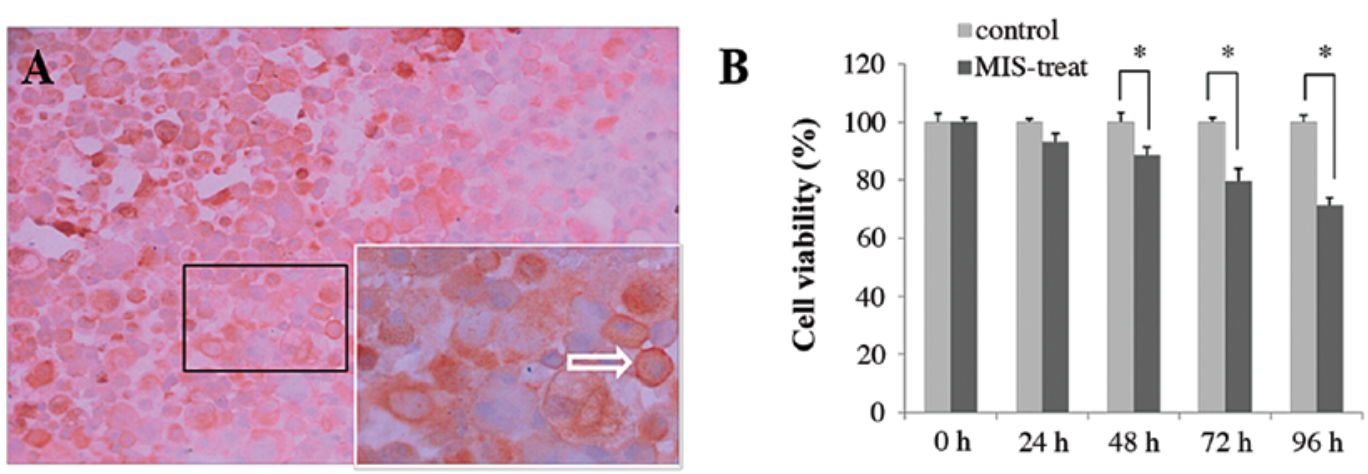

C
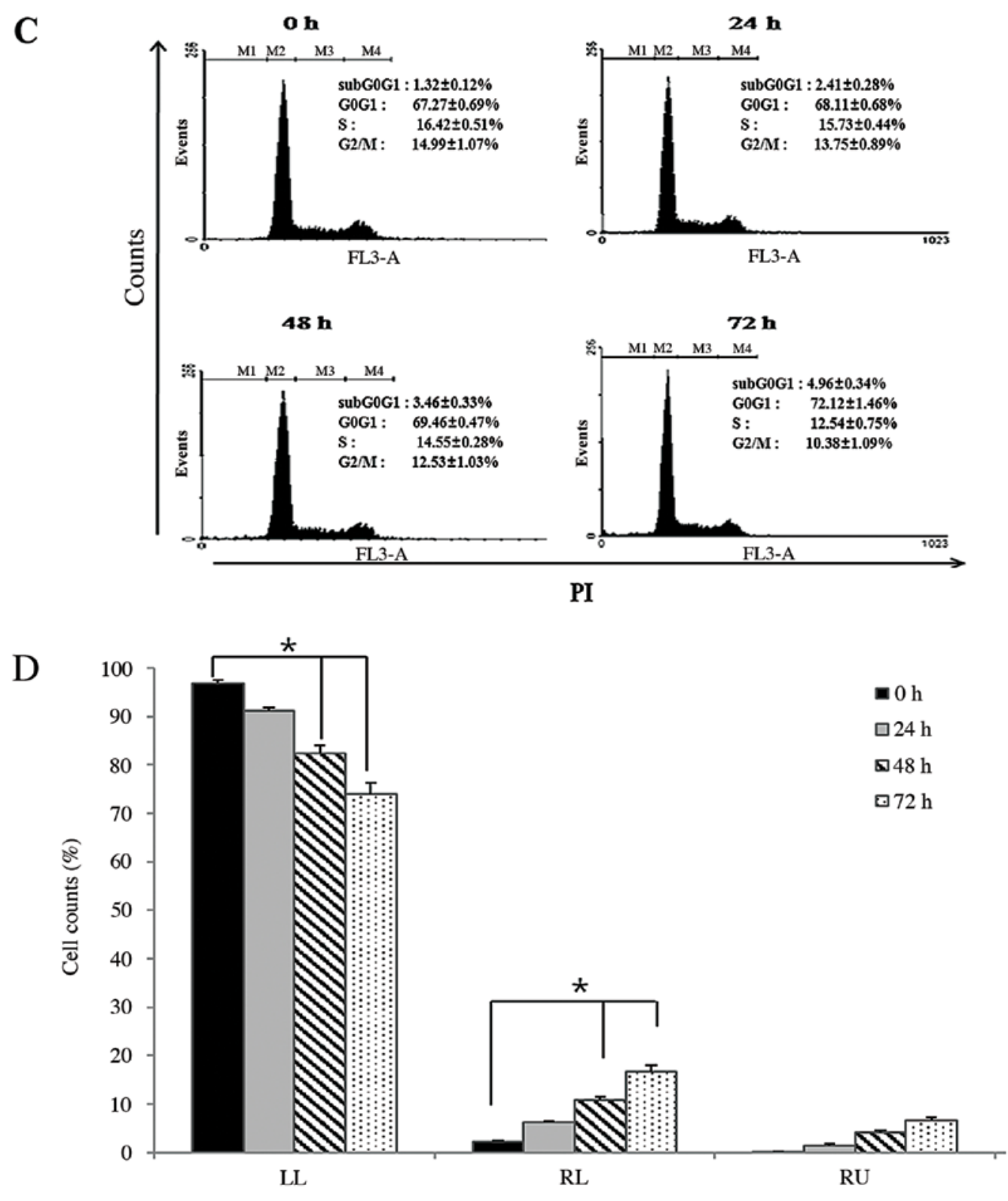

Figure 1. (A) Expression of MISRII on AN3CA endometrial cancer cells as assessed by immunocytochemistry using an MISRII antibody. AN3CA cells showed strong specific MISRII staining, which was the most intense at the cell surface (x200). At higher magnification (x400) the arrow in the boxed area shows strong surface expression of MISRII on AN3CA cell (characteristic of $n=3$ ). (B) Inhibition of cell growth by MIS in AN3CA cells was assessed by the cell viability MTT assay. AN3CA cells were treated with $10 \mu \mathrm{g} / \mathrm{ml}(71.4 \mathrm{nM})$ MIS for 0, 24, 48, 72 and $96 \mathrm{~h}$, stained with MTT, and the absorbance was read at $550 \mathrm{~nm}$. Results are presented as a percentage of the untreated control which was calculated as mean absorbance of treated cells/mean absorbance of control cells $x 100$ and expressed as mean \pm standard deviation (SD) from three independent experiments ( $\mathrm{P}<0.05$ as compared to corresponding control cells). (C) To determine cell cycle distribution after exposure to MIS, AN3CA endometrial cancer cells were treated with $10 \mu \mathrm{g} / \mathrm{ml} \mathrm{MIS} \mathrm{for} 0,24,48$ and $72 \mathrm{~h}$, trypsinized and fixed in $100 \%$ methanol. Then washed cells were exposed to propidium iodide/RNase solution and histograms of cellular DNA content obtained by flow cytometry (representative of 3 replicates). (D) To detect induction of apoptosis by MIS, AN3CA endometrial cancer cells were treated with $10 \mu \mathrm{g} / \mathrm{ml} \mathrm{MIS}$ for $0,24,48$ and $72 \mathrm{~h}$, respectively, and externalization of phosphatidylserine assessed by measuring Annexin V-FITC binding using propidium iodide as a counterstain (LL, surviving cells; RL, early apoptotic cells; RU, late apoptotic and necrotic cells, ${ }^{\mathrm{P}}<0.05$ as compared to corresponding $0 \mathrm{~h}$, representative of 3 replicates). 
A

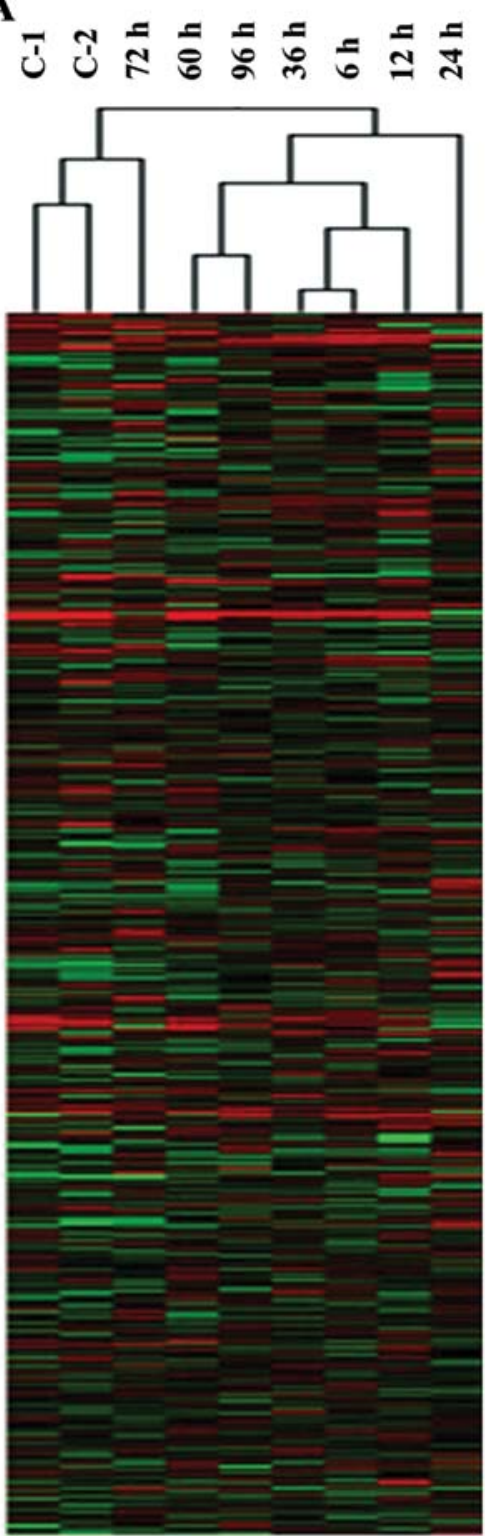

B
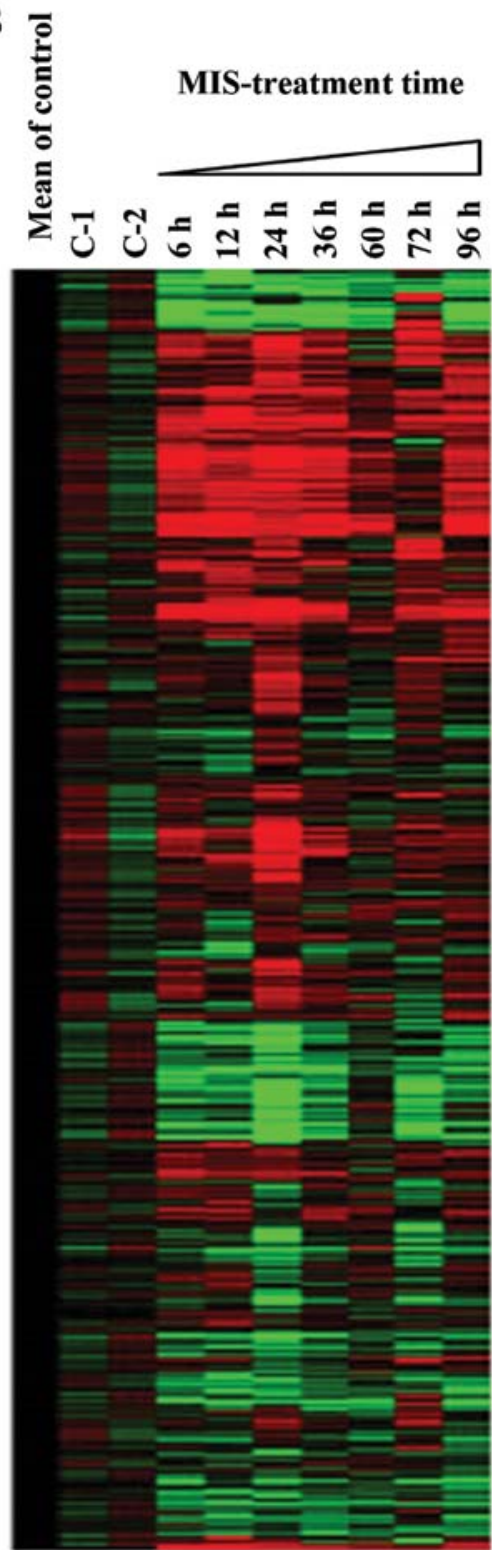

Figure 2. Differential gene expression profiling and identification of molecular changes in the AN3CA cells that were treated with $10 \mu \mathrm{g} / \mathrm{ml}$ MIS are shown by (A) unsupervised hierarchical clustering of 11,470 genetic elements with minimum selection and filtering criteria. (B) Mathematical comparisons of genes between $0 \mathrm{~h}$ and the 6-96 $\mathrm{h}$ time points. Selected 2,688 genes showing at least 1.3-fold changes induced by MIS treatment compared to non-treated control $(0 \mathrm{~h})$ are visualized as a heat map where red are higher, and green are lower than the mean value of non-treated cells.

Encyclopedia of Genes and the Genomes (KEGG) pathway database (http://www.genome.jp/kegg/) which maps genes to known pathways and provides a summary of the biological processes affected. Based on this database analysis, we identified 20 pathways containing at least 10 genetic elements from the 2,668 differentially expressed genes. AN3CA cells treated with MIS fall into cancer-related signaling pathways involving Wnt, cell cycle, MAPK, p53 and apoptosis pathways (Table I). From these, genes associated with Wnt, cell cycle regulation and apoptosis pathways were depicted as heat maps (Fig. 3A). In Wnt signaling pathways, CTNNBIP1 and CXXC4 genes were upregulated and JUN was downregulated compared to non-treatment. CDK2 gene was downregulated in cell cycle pathways. In apoptosis pathways, the APAF1 gene was upregulated while caspase-3 was slightly downregulated (Fig. 3A).
Differentially expressed genes in AN3CA cells, that were examined by western analysis include phospho-c-Jun, ICAT, IDAX, CDK2, p130, p107, APAF-1, caspase-3 and PARP, which confirmed upregulation of ICAT and IDAX and downregulation of phospho-c-Jun in Wnt signaling pathways, downregulation of CDK2 and slight upregulation of p130 and p107 in cell cycle pathways, and upregulation of APAF- 1 and cPARP and downregulation of full length caspase- 3 in the apoptosis pathway (Fig. 3B).

\section{Discussion}

In previous studies, MIS has been shown to inhibit the growth of ovarian, cervical and endometrial cancer cell lines. Treatment with recombinant human MIS induced upregulation of both p107 and p130 in non-HPV-related cervical cancer 


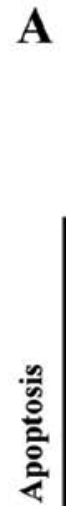
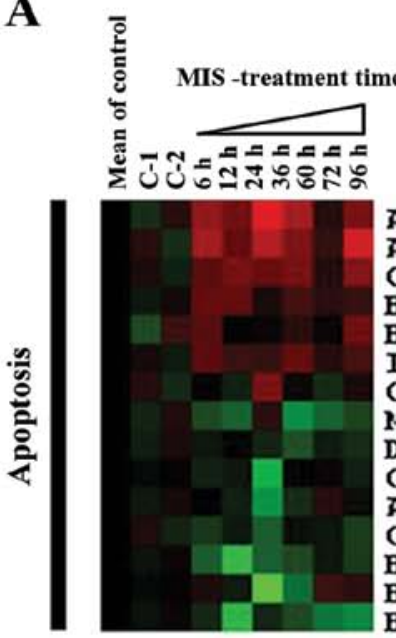

APAF 1

ATR

CFLAR

BAD

BID

IRAK2

CASP?

MAP 3K14

DFF

CASP3

ATFi 1

CASP9

BIRC2

BAX

BIRC2

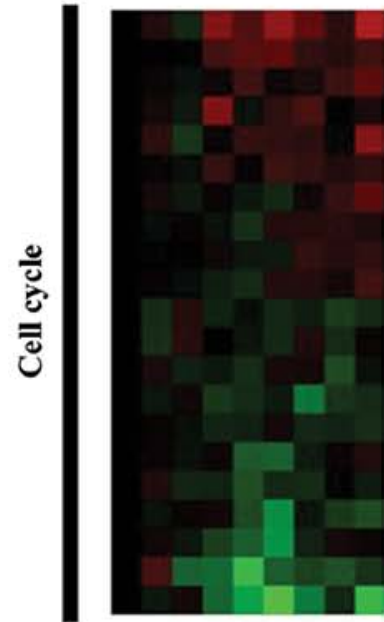

ATM

CDKA2

E2F1

CDC14B

CDC?

AHAPC4

CHEK2

DARAPC?

CDC25C

CDKH2B

GADD45A

ABL 1

MCA2

GRDD45B

MCA5

MCA4

CCIE1 1

CDC25

CDK2

CCrE2 2

CDKN2C

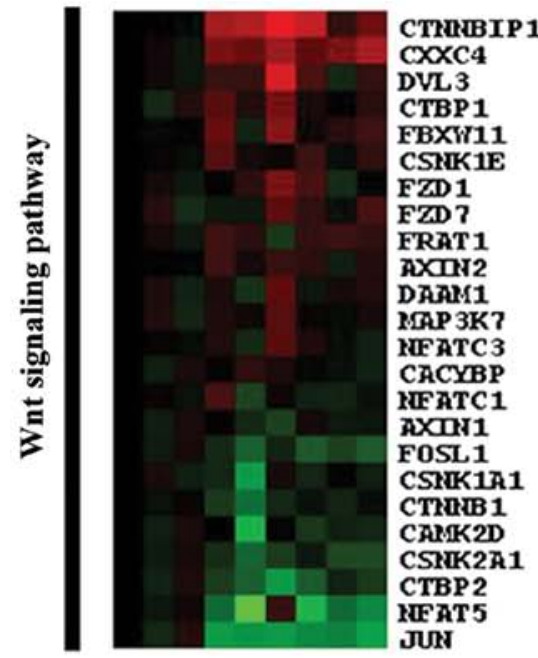

B
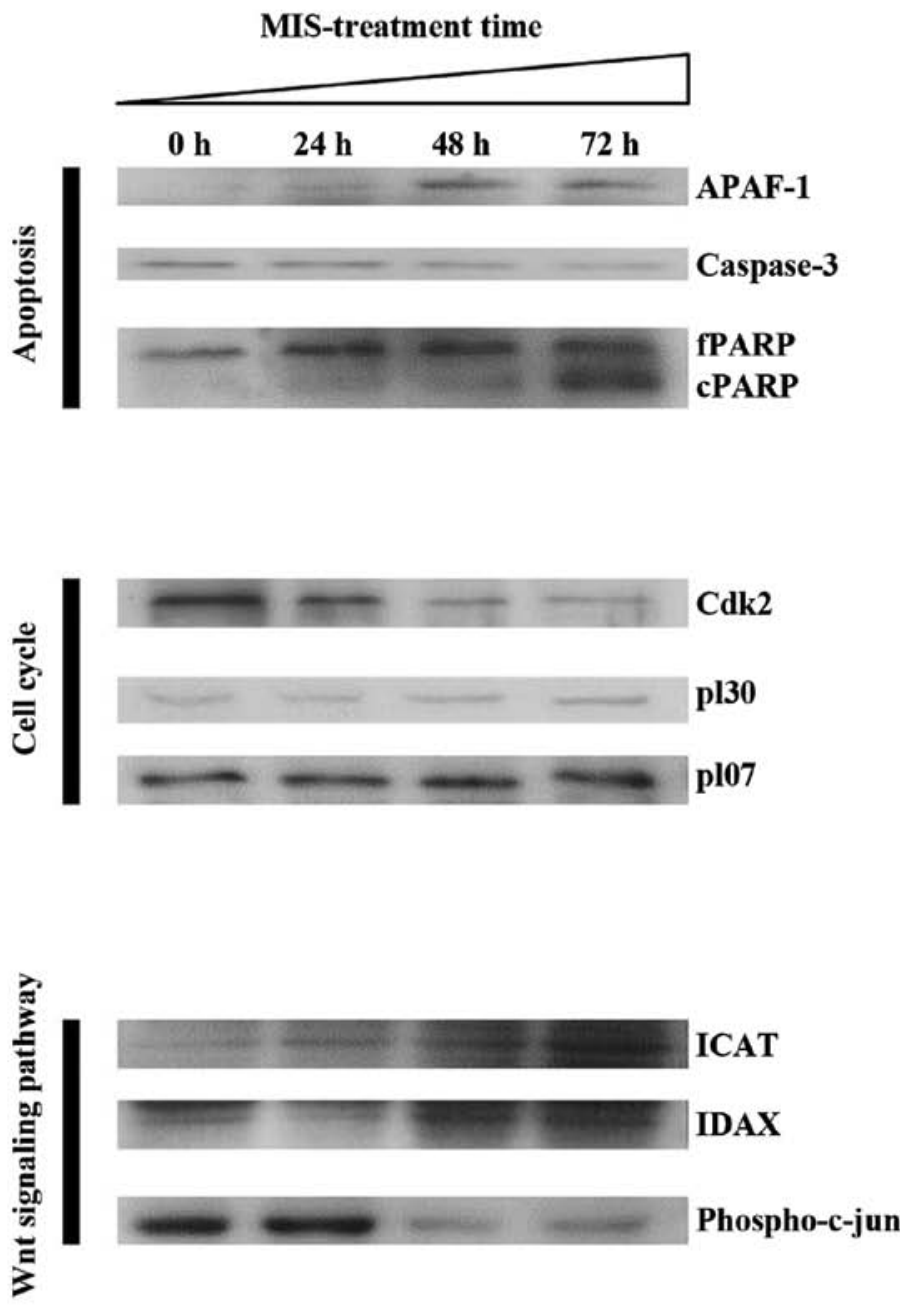

Actin

Figure 3. (A) Selective expression profile of KEGG pathway-related genetic elements retrieved from 2,688 differentially expressed genes. Fifteen genes were mapped to apoptosis, 21 to cell cycle and 24 to Wnt signaling pathways in the KEGG database. These elements were then visualized as a heat map of expression of genetic elements where red are higher, and green are lower than the mean value of non-treated cells. (B) Western blot analysis for the expression of apoptosis, cell cycle and Wnt signaling pathway-related proteins in AN3CA cells treated with $10 \mu \mathrm{g} / \mathrm{ml}$ MIS for 0,24, 48 and $72 \mathrm{~h}$, representative of 3 replicates.

cell lines (13) and inhibited the growth of the ovarian cancer cell line through induction of the CDK inhibitor p16 at the protein level and downregulation of p130 and upregulation of $\mathrm{E} 2 \mathrm{~F} 1$ when $\mathrm{Rb}$ levels were not detectable (14). In endometrial cancer cell lines (AN3CA) lacking p16 and pRb, MIS-induced apoptosis was associated with an increase in p107 and p130 and a decrease in E2F1 expression at $72 \mathrm{~h}$ (10). Thus, gene regulation by MIS appears to be specific to tumor type, but in these studies involves regulation of cell cycle pathways.

The cornerstone of curative therapy for endometrial cancer is surgical treatment, including complete hysterectomy, adnexectomy and appropriate surgical staging in patients 
Table I. Summary of the KEGG pathways that are part of the characteristic molecular signature of MIS in AN3CA cells.

\begin{tabular}{|c|c|c|c|}
\hline KEGG pathway & Gene counts & Upregulated genes & Downregulated genes \\
\hline Pathways in cancer & 41 & 17 & 24 \\
\hline MAPK signaling pathway & 35 & 17 & 18 \\
\hline Alzheimer's disease & 23 & 12 & 11 \\
\hline Regulation of actin cytoskeleton & 23 & 7 & 16 \\
\hline Ubiquitin mediated proteolysis & 21 & 14 & 7 \\
\hline Focal adhesion & 21 & 4 & 17 \\
\hline Wnt signaling pathway & 20 & 8 & 12 \\
\hline $\mathrm{T}$ cell receptor signaling pathway & 17 & 6 & 11 \\
\hline GnRH signaling pathway & 16 & 8 & 8 \\
\hline Chronic myeloid leukemia & 15 & 4 & 11 \\
\hline Axon guidance & 14 & 6 & 8 \\
\hline Colorectal cancer & 14 & 4 & 10 \\
\hline Huntington's disease & 14 & 5 & 9 \\
\hline ErbB signaling pathway & 14 & 4 & 10 \\
\hline Prostate cancer & 14 & 4 & 10 \\
\hline Apoptosis & 14 & 7 & 7 \\
\hline Pathogenic Escherichia coli infection & 14 & 1 & 13 \\
\hline Cell cycle & 13 & 4 & 9 \\
\hline p53 signaling pathway & 11 & 4 & 7 \\
\hline Tight junction & 11 & 4 & 7 \\
\hline
\end{tabular}

There were significant pathways including more than 10 genes by one pathway in outliers of 2,688 genes which showed expression more than 1.3 -fold increase or decrease.

considered at high risk. The type of surgery and postoperative therapy depends on the stage and other clinicopathological risk factors and survival is strongly dependent on surgical stage. Recent advances in the understanding of the molecular and genetic basis of endometrial cancer have led to the development of targeted therapies that inhibit angiogenesis and the cellular signaling pathways involved in cell growth and proliferation. Trastuzumab, a human EGFR type II (HER2)related inhibitor that affects signal transduction, is currently a standard treatment for HER2-positive breast cancer. Certain serous adenocarcinomatous endometrial cancers which are characterized by overexpression of HER 2 could be helped by trastuzumab (15). mTOR inhibition with temsirolimus has encouraging single-agent activity in endometrial cancer which has been shown to be more effective in chemotherapynaive patients than in chemotherapy-treated patients and is independent of PTEN status (16). Another mTOR inhibitor, ridaforolimus, has antitumor activity and acceptable tolerability in advanced endometrial cancer patients (17). Given the potential of MIS as a therapeutic for endometrial cancer, particularly the more aggressive Type II, it is important to understand the genes regulated by MIS.

To demonstrate the molecular mechanism by which MIS might inhibit the growth of an endometrial carcinoma cell line, we first evaluated the expression of MISRII on the cell surface of the endometrial carcinoma cell line, AN3CA. After confirmation of the MISRII expression on the AN3CA by immunohistochemistry, we examined the inhibitory effect of
MIS on the growth of the endometrial cell line and showed $7-29 \%$ reduction in cell survival, an increase of cellular apoptosis and a small change in cell cycle parameters. Microarray and western blot analyses confirmed changes in pathways related to cell cycle, apoptosis and Wnt signaling.

For example, ICAT, the protein encoded by CTNNBIP1, a negative regulator of the Wnt signaling pathway (18) binds CTNNB1 ( $\beta$-catenin) and prevents its intracellular interaction with TCF (T-cell transcription factor) family members. IDAX, the protein encoded by the $\mathrm{CXXC} 4$ gene also functions as a negative regulator of the Wnt signaling pathway by directly binding to the PDZ domain of Disheveled (DVL) in the nucleus (19). C-Jun transcribes a protein that is required for progression through the G1 phase of the cell cycle as c-Jun null cells show increased G1 arrest (20). C-Jun is also a target gene of $\beta$-catenin, which, if decreased, could result in tumor suppression, indicating that MIS could possibly result in tumor suppression, through regulation of Wnt signaling.

APAF1 encodes a cytoplasmic protein that forms one of the central hubs in the apoptosis regulatory network, and its activation of PARP induces necrotic and programmed cell death, thus supporting the most robust changes seen in these studies (Fig. 1D, i.e., apoptosis). In previous studies, MIS has been documented to increase both p107 and p130 in AN3CA cells (10), which was also correlated with inhibition of proliferation in cervical cancer cell lines (13). Slight upregulation of p107 and p130 was shown in the study. However, other genes associated with cell cycle demonstrated significant differences 
after MIS treatment; in cell cycle pathways, CDK2 is essential for the G1/S transition and reduction of CDK2 levels correlates with cell cycle arrest.

In summary, these findings indicate that MIS regulates the proliferation of tumor cells through modulation of apoptosis and cell cycle pathways, and by causing blockade of the Wnt/ $\beta$-catenin signaling pathways. The findings support the hypothesis that MIS was able to function as a potential therapeutic for endometrial cancer by targeting these molecular pathways.

\section{References}

1. Nicolaije KA, Ezendam NP, Vos MC, Boll D, Pijnenborg JM, Kruitwagen RF, Lybeert ML and van de Poll-Franse LV: Follow-up practice in endometrial cancer and the association with patient and hospital characteristics: A study from the populationbased PROFILES registry. Gynecol Oncol 129: 324-331, 2013.

2. Bokhman JV: Two pathogenetic types of endometrial carcinoma Gynecol Oncol 15: 10-17, 1983.

3. Dong P, Kaneuchi M, Konno Y, Watari H, Sudo S and Sakuragi N Emerging therapeutic biomarkers in endometrial cancer. Biomed Res Int 2013: 130362, 2013.

4. Slomovitz BM and Coleman RL: The PI3K/AKT/mTOR pathway as a therapeutic target in endometrial cancer. Clin Cancer Res 18 : 5856-5864, 2012.

5. Matias-Guiu X and Prat J: Molecular pathology of endometrial carcinoma. Histopathology 62: 111-123, 2013.

6. Acharya S, Hensley ML, Montag AC and Fleming GF: Rare uterine cancers. Lancet Oncol 6: 961-971, 2005.

7. Bakkum-Gamez JN, Aletti G, Lewis KA, Keeney GL, Thomas BM, Navarro-Teulon I and Cliby WA: Müllerian inhibiting substance type II receptor (MISIIR): A novel, tissuespecific target expressed by gynecologic cancers. Gynecol Oncol 108: 141-148, 2008.

8. Hwang SJ, Suh MJ, Yoon JH, Kim MR, Ryu KS, Nam SW, Donahoe PK, Maclaughlin DT and Kim JH: Identification of characteristic molecular signature of Müllerian inhibiting substance in human HPV-related cervical cancer cells. Int J Oncol 39: 811-820, 2011
9. Nam SW, Jo YS, Eun JW, Song JY, Ryu KS, Lee JY, Lee JM, MacLaughlin DT and Kim JH: Identification of large-scale characteristic genes of Müllerian inhibiting substance in human ovarian cancer cells. Int J Mol Med 23: 589-596, 2009.

10. Renaud EJ, MacLaughlin DT, Oliva E, Rueda BR and Donahoe PK: Endometrial cancer is a receptor-mediated target for Müllerian Inhibiting Substance. Proc Natl Acad Sci USA 102: 111-116, 2005.

11. Masiakos PT, MacLaughlin DT, Maheswaran S, et al: Human ovarian cancer, cell lines, and primary ascites cells express the human Müllerian inhibiting substance (MIS) type II receptor, bind, and are responsive to MIS. Clin Cancer Res 5: 3488-3499, 1999.

12. Lorenzo HK, Teixeira J, Pahlavan N, Laurich VM, Donahoe PK and MacLaughlin DT: New approaches for high-yield purification of Müllerian inhibiting substance improve its bioactivity. J Chromatogr B Analyt Technol Biomed Life Sci 766: 89-98, 2002.

13. Barbie TU, Barbie DA, MacLaughlin DT, Maheswaran S and Donahoe PK: Müllerian Inhibiting Substance inhibits cervical cancer cell growth via a pathway involving p130 and p107. Proc Natl Acad Sci USA 100: 15601-15606, 2003.

14. Ha TU, Segev DL, Barbie D, et al: Müllerian inhibiting substance inhibits ovarian cell growth through an $\mathrm{Rb}$-independent mechanism. J Biol Chem 275: 37101-37109, 2000.

15. Elsahwi KS and Santin AD: erbB2 overexpression in uterine serous cancer: A molecular target for trastuzumab therapy. Obstet Gynecol Int 2011: 128295, 2011.

16. Oza AM, Elit L, Tsao MS, et al: Phase II study of temsirolimus in women with recurrent or metastatic endometrial cancer: A trial of the NCIC Clinical Trials Group. J Clin Oncol 29: 3278-3285, 2011.

17. Colombo N, McMeekin DS, Schwartz PE, et al: Ridaforolimus as a single agent in advanced endometrial cancer: Results of a single-arm, phase 2 trial. Br J Cancer 108: 1021-1026, 2013.

18. Baarsma HA, Königshoff $M$ and Gosens R: The WNT signaling pathway from ligand secretion to gene transcription: Molecular mechanisms and pharmacological targets. Pharmacol Ther 138: 66-83, 2013.

19. Hino S, Kishida S, Michiue T, Fukui A, Sakamoto I, Takada S, Asashima $\mathrm{M}$ and Kikuchi A: Inhibition of the Wnt signaling pathway by Idax, a novel Dvl-binding protein. Mol Cell Biol 21: 330-342, 2001.

20. Wisdom R, Johnson RS and Moore C: c-Jun regulates cell cycle progression and apoptosis by distinct mechanisms. EMBO J 18: 188-197, 1999. 